\title{
Auxin influx carriers stabilize phyllotactic patterning
}

\author{
Katherine Bainbridge, ${ }^{1}$ Soazig Guyomarc' $h,{ }^{1}$ Emmanuelle Bayer, ${ }^{1}$ Ranjan Swarup, ${ }^{2}$ \\ Malcolm Bennett, ${ }^{2}$ Therese Mandel, ${ }^{1}$ and Cris Kuhlemeier ${ }^{1,3}$ \\ ${ }^{1}$ Institute of Plant Sciences, University of Bern, Altenbergrain 21, 3013 Bern, Switzerland; ${ }^{2}$ University of Nottingham, \\ NG7 2RD Nottingham, United Kingdom
}

\begin{abstract}
One of the most striking features of plant architecture is the regular arrangement of leaves and flowers around the stem, known as phyllotaxis. Peaks in concentration of the plant hormone auxin, generated by the polar localization of the PIN1 auxin efflux carrier, provide the instructive signal for primordium initiation. This mechanism generates the spacing between neighboring primordia, which results in regular phyllotaxis. Studies of the role of auxin transport in phyllotactic patterning have focused on PIN1-mediated efflux. Recent computer simulations indicate an additional role for transporter-mediated auxin uptake. Mutations in the AUX1 auxin influx carrier have not, however, been reported to cause an aerial phenotype. Here, we study the role of AUX1 and its paralogs LAX1, LAX2, and LAX3. Analysis of the quadruple mutant reveals irregular divergence angles between successive primordia. A highly unusual aspect of the phenotype is the occurrence of clusters of primordia, in violation of classical theory. At the molecular level, the sharp peaks in auxin levels and coordinated PIN polarization are reduced or lost. In addition, the increased penetrance of the phenotype under short-day conditions suggests that the AUX LAX transporters act to buffer the PIN-mediated patterning mechanism against environmental or developmental influences.
\end{abstract}

[Keywords: AUX1 LAX genes; PIN1; auxin; phyllotaxis; meristem; leaf development]

Supplemental material is available at http://www.genesdev.org.

Received November 5, 2007; revised version accepted January 9, 2008.

The ability of plants to initiate organs separated by regular angles at the shoot meristem results in the generation of the beautiful and diverse phyllotactic patterns found in nature. The understanding of the mechanisms underlying phyllotaxis is one of the classical problems in botany and has provided inspiration for numerous experimental and theoretical studies. In 1868, Wilhelm Hofmeister published his careful observations of lateral organ initiation in a large number of plant meristems. In this work he noted that, irrespective of the phyllotactic pattern, a new organ primordium is always initiated at a maximal distance from previously formed primordia (Hofmeister 1868). Following over a century of research, this key observation still provides the foundation for all models of phyllotactic patterning (Smith et al. 2006b; Kuhlemeier 2007).

The most prevalent phyllotactic pattern found in nature is spiral phyllotaxis, as exhibited by Arabidopsis, in which successively initiated primordia are separated by an average divergence angle of $137.5^{\circ}$ (Kuhlemeier 2007). Recently, the plant hormone auxin has been found to provide the inductive signal in primordium initiation

${ }^{3}$ Corresponding author.

E-MAIL cris.kuhlemeier@ips.unibe.ch; FAX 41-31-332-20-59.

Article is online at http://www.genesdev.org/cgi/doi/10.1101/gad.462608. and phyllotactic patterning (Reinhardt et al. 2000). Peaks in expression of the synthetic auxin-signaling reporter $D R 5:$ GFP correlate with the sites of incipient primordium formation (Benkova et al. 2003; Heisler et al. 2005; Smith et al. 2006a). At the meristem, the PIN1 auxin efflux carrier is predominantly expressed in the outermost L1 layer (Reinhardt et al. 2003). The protein is polarly localized at the plasma membrane, forming convergence points that correspond to sites of auxin maxima and subsequent organ outgrowth (Benkova et al. 2003; Reinhardt et al. 2003; de Reuille et al. 2006; Smith et al. 2006a). In computer models simulating phyllotactic patterning, PIN1 is predicted to polarize toward the neighboring cell with the highest auxin concentration (Jonsson et al. 2006; Smith et al. 2006a). This positive feedback loop should result in accumulation of auxin in defined groups of cells, until levels are sufficient to trigger organ development. Simultaneously, auxin is drained from neighboring cells, resulting in levels below that required for primordium initiation.

Auxin, as visualized by DR5::GFP, is restricted to the L1 (Smith et al. 2006a). This restriction is thought to be mediated by the AUX1 import protein, which shows specific L1 localization and could facilitate auxin accumulation in the epidermis (Reinhardt et al. 2003; Heisler and Jonsson 2006). Current computer simulations of 
phyllotaxis only consider the L1 cell layer of the meristem (de Reuille et al. 2006; Jonsson et al. 2006; Smith et al. 2006a) and therefore do not model the possible function of AUX1 in restricting diffusion of auxin into subdermal layers. In omitting both sub-L1 layers and auxin importer activity, computer models of phyllotaxis may be missing a vital role for these proteins in concentrating auxin within cells and preventing diffusion from the L1, which could be critical for phyllotactic patterning (Heisler and Jonsson 2006).

The chemiosmotic model of auxin transport predicts that a certain amount of influx can occur even in the absence of import carriers (Kramer and Bennett 2006). The presence of influx carriers could prove critical in cells where rapid uptake is essential to maintain gradients, which would otherwise be lost owing to apoplastic diffusion (Marchant et al. 1999; Swarup et al. 2001, 2005; Kramer and Bennett 2006). Indeed, the gravitropic response in roots requires specific $A U X 1$ expression in the lateral root cap and epidermal cells in order for the auxin gradients required for root curvature to be established (Swarup et al. 2005). Computer models additionally predict that carrier-mediated auxin uptake is crucial in maintaining the high auxin concentration within the vasculature in tissues with small cell size (Kramer 2004). In addition, models suggest that expression of AUX1 could stabilize auxin peaks generated by PIN1 transport (Heisler and Jonsson 2006).

The Arabidopsis genome encodes four putative auxin influx carriers: AUX1 and the LAX1, LAX2, and LAX3 (Like AUX1) genes (Parry et al. 2001). The AUX1 and LAX3 proteins are plasma membrane-localized and have been shown to actively transport auxin into cells (Swarup et al. 2004, 2008; Yang et al. 2006). AUX1 and the three $L A X$ genes exhibit between $73 \%$ and $82 \%$ similarity at the amino acid level, suggesting a conserved function in auxin uptake (Parry et al. 2001). No aerial phenotype has previously been reported for aux1 or for the lax single mutants. A role for active auxin influx in controlling the initiation of primordia at the shoot meristem was, however, suggested in experiments with the pin1 aux1 double mutant (Reinhardt et al. 2003). In these experiments, localized auxin application to the pin1 aux1 meristem resulted in wider primordia, compared with the single pin 1 mutant background. In addition, tomato apices treated with auxin influx inhibitors initiated wider primordia than control apices (Stieger et al. 2002). Although computer models suggest that AUX1 LAX proteins could be crucial for developmental patterning, until now the biological evidence for this role has been limited to the involvement of AUX1 and LAX3 in root development (Bennett et al. 1996; Marchant et al. 1999, 2002; Swarup et al. 2001, 2005, 2008; Kramer 2004, 2006; Heisler and Jonsson 2006; De Smet et al. 2007).

In this study, we report the characterization of the phyllotactic phenotype of the aux1 and lax mutants. We show that double, triple, and quadruple mutant combinations result in disorganized phyllotactic patterning, with primordia initiating at aberrant angles at the shoot apical meristem. Most strikingly, primordia often formed in close proximity to each other in aux1 lax mutants, thereby violating Hofmeister's Rule. The disorganized phyllotactic pattern correlates with a loss of defined peaks in auxin reporter signal and decreased frequency and altered spacing of PIN1 convergence points. We hypothesize that AUX1 LAX function aids the coordination of PIN1 polarization and the creation of defined auxin maxima leading to primordium initiation in a regular and stable phyllotactic pattern, especially under adverse environmental conditions.

\section{Results}

Mutations in the Aux1 Lax auxin influx carrier genes result in altered phyllotactic patterning at the vegetative meristem

In order to investigate the effects of mutations in the AUX1 LAX auxin influx carriers on phyllotactic patterning, an aux1 lax1 lax2 lax3 quadruple mutant (referred to as "quad") was created. We first investigated the effects of loss of AUX1 LAX activity on the aerial phenotype of long-day-grown plants (Fig. 1A,B). The most immediately apparent phenotype in the quad was the narrow and downwardly rolled leaves, which showed lefthanded helical twisting; however, no phyllotactic phenotype was evident at the vegetative stage under longday conditions (Fig. 1A,B).

Under long-day conditions, Arabidopsis makes only a few leaves and rapidly switches to flowering. In order to prolong the vegetative period, we grew plants under short-day conditions and followed the sequence of leaf initiation from germination to floral transition. In wildtype plants, we found that the average angle between successive leaves was very close to the theoretical divergence angle of $137.5^{\circ}$. (Fig. 1C,G; Kuhlemeier 2007). During early development, the spiral phyllotaxis of the quad was unaffected in the majority of plants, with a divergence angle very similar to that seen in wild type (Fig. $1 \mathrm{C}, \mathrm{D}, \mathrm{G})$. Later in development, quad plants exhibited a clear phyllotactic phenotype. This was distinct from the twisting phenotype that was first seen in leaves of $\sim 1.5$ $\mathrm{cm}$ in length. The phyllotactic phenotype was defined by two key features: Firstly, the divergence angle between successively formed leaves became increasingly irregular, with leaves frequently initiating at much wider or narrower angles than in wild-type plants, and sometimes reversing the direction of the ontogenetic spiral (Fig. 1DF). Secondly, periods of arrest in macroscopically visible leaf formation occurred (Fig. 1E; Supplemental Fig. S1). The precise time point of arrest was not simultaneous in all quad plants (Supplemental Fig. S1), but was observed in the first plants at $\sim 35 \mathrm{~d}$ after germination (DAG). Owing to the lack of a phyllotaxis phenotype in the aux1 lax single mutants (data not shown), initial analysis concentrated on the clear phenotype exhibited by the quad mutant.

The variation in the time of arrest, and the irregular divergence angles between successively formed leaves in the quad, led to phenotypic diversity in plants observed 

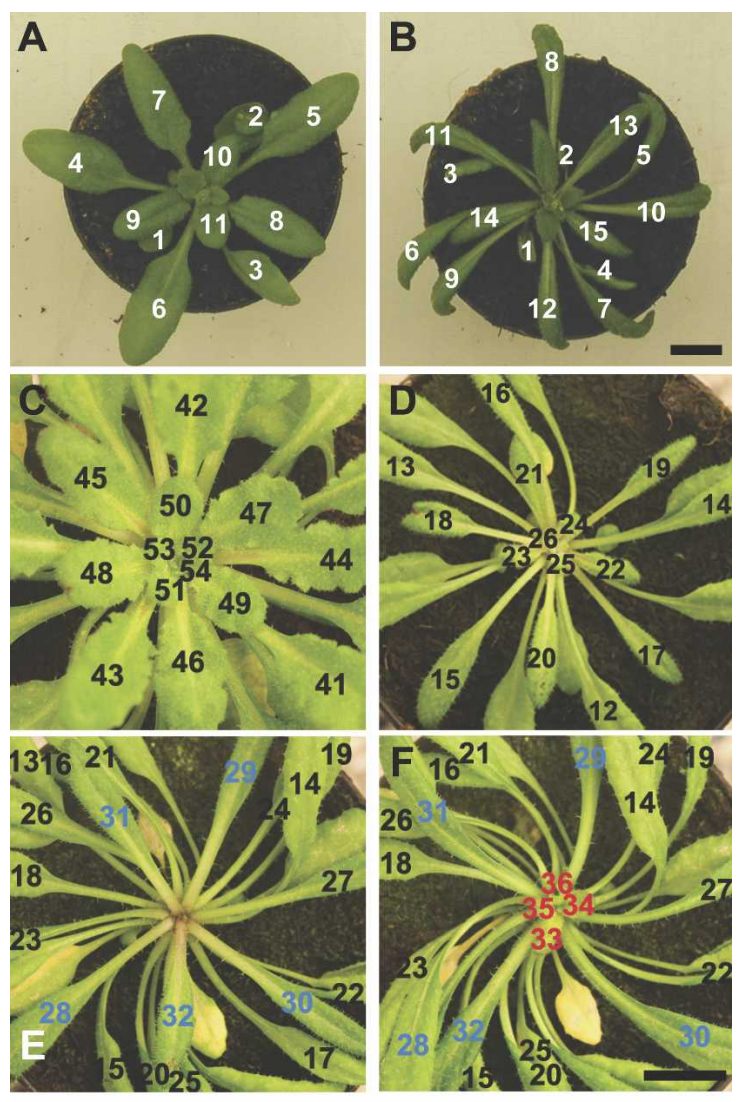

G

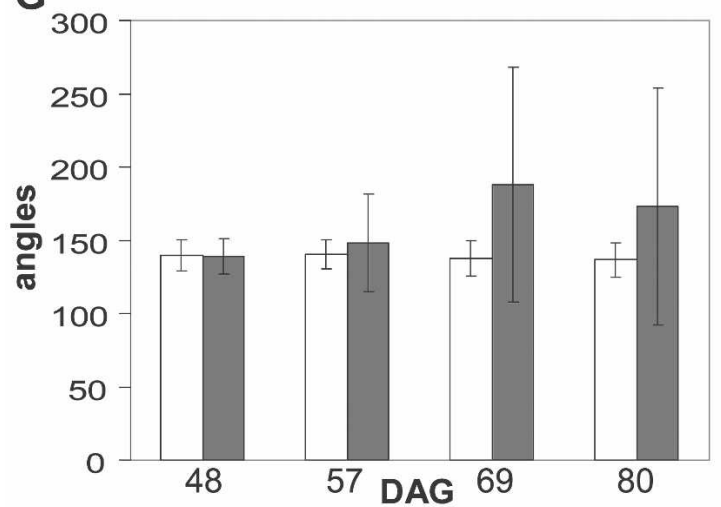

Figure 1. Leaf initiation in wild-type and quadruple aux1 lax mutant plants. Photographs showing vegetative rosettes of wild-type and quad plants under long- and short-day conditions. (A) Wild type; long days. (B) quad; long days showing narrow, twisted leaf blades and regular, spiral phyllotaxis. $(C)$ Wild type; short days, 73 DAG. $(D-F)$ The same short-day-grown quad plant at three different time points. (D) quad 62 DAG. (E) quad 73 DAG. $(F)$ quad 78 DAG. Leaves numbered in order of initiation. (Blue) Leaves initiated immediately before arrest; (red) leaves initiated after arrest. Bars, $1 \mathrm{~cm}$. (G) Graph showing the mean divergence angle between successively formed leaves at four different time points. (White bars) quad; (gray bars) wild type. Error bars represent the standard error of the mean. $n=20$.

at the same time point. Arrest in organ formation occurred in all quad plants $(n=40)$ before floral transition, but was never seen in wild type. This arrest was, how- ever, temporary, and following a lag period, newly formed organs were once more visible at the apex (Fig. 1F; Supplemental Fig. S1). Periods of arrest resulted in a reduction in the average number of leaves formed in the quad relative to wild-type plants during this stage of growth (Supplemental Fig. S2). Before arrest, the rate of leaf initiation was comparable in wild-type and quad plants, arguing against a general increase in plastochron length until the point when arrest occurs (Supplemental Fig. S2, see 45 DAG).

In order to determine whether this observed irregularity in phyllotaxis in quad plants occurs at the point of ontogeny or as a result of shifts in leaf positioning following initiation, primordium formation at the meristem was examined using low-vacuum scanning electron microscopy. In wild-type plants, the angle between successive organs visible at the vegetative apical meristem approximated $137.5^{\circ}$ (Fig. 2A). In contrast, quad mutants exhibited a dramatic alteration in the divergence angle between leaf primordia at the meristem, with primordia initiating at much narrower or at wider
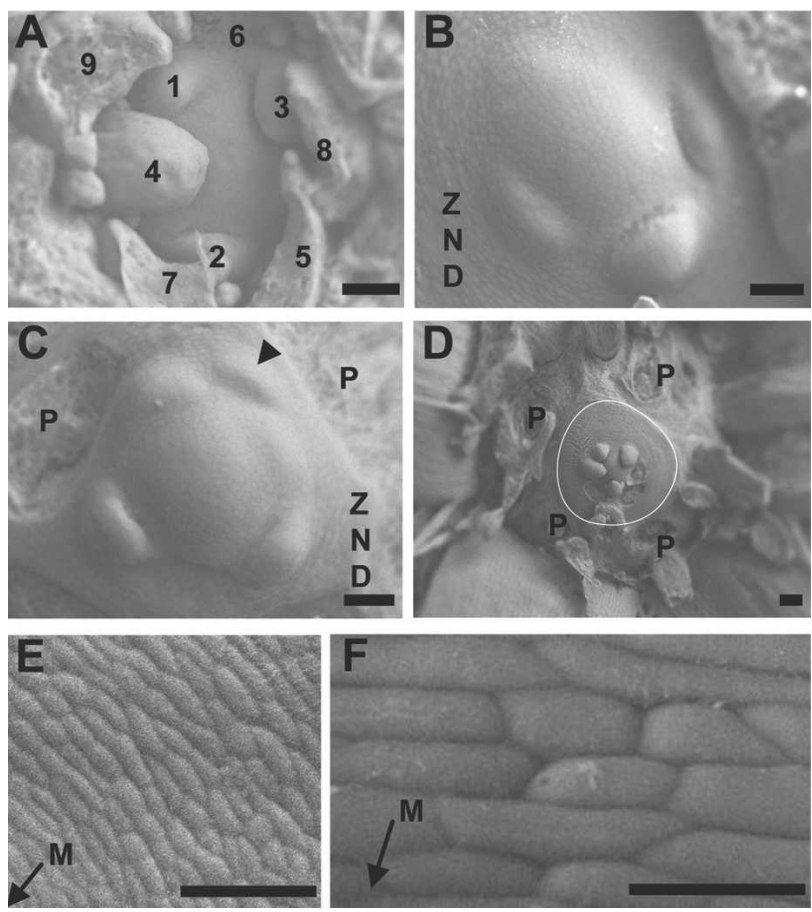

Figure 2. Irregular primordium initiation at vegetative meristems of the quadruple aux1 lax mutant. Transversal scanning electron microscope images of wild-type and quad meristems. $(A)$ Wild type. $(B-F)$ quad. Leaf primordia are labeled in the order of initiation, with 1 denoting the youngest visible primordium. In cases where the order of initiation is unclear, primordia are not numbered. $\mathrm{P}$ denotes the base of primordia removed during dissection. The arrowhead in $C$ indicates an abortive primordium that has initiated and moved away from the meristem without developing further. The white line in $D$ outlines the periphery of the ZND. $(E, F)$ Cells of the ZND. The arrows indicate the direction of the meristem (M). Bars: $A-C, E, 50 \mu \mathrm{m} ; D$, $100 \mu \mathrm{m} ; F, 20 \mu \mathrm{m}$. Plants dissected at 55 DAG. 
angles than the wild type (Fig. 2B,C). Notably, primordia were often seen to arise in clusters, with no bulges around the rest of the meristem (Fig. 2B).

In some cases, initiated primordia were observed to have moved away from the meristem without undergoing development beyond the initial stage of bulging (Fig. $2 \mathrm{C}$, arrowhead). In the absence of primordium formation, cell division at the meristem appeared to continue, pushing out cells into the peripheral zone despite the lack of bulging. This resulted in the formation of rings of undifferentiated cells, which were never seen in wild-type plants (Fig. 2B-F). This zone of no distinction (ZND) varied in size in different plants, but often formed an extensive area around the meristem (Fig. 2D). The cells in the ZND, which presumably followed a default pathway in the absence of an appropriate signal to differentiate and become incorporated into primordia, were circumferentially elongated and formed ordered rings around the meristem (Fig. 2E,F). At the point of initiation, primordia were a similar size to those of wild-type plants, suggesting that primordium size regulation and primordium spacing can be uncoupled.

At the quad inflorescence meristem, similar irregularities were seen in phyllotactic patterning (Supplemental Fig. S3). These irregularities were more severe under short days, but also evident under a long photoperiod (Supplemental Fig. S3; data not shown). Many of the floral primordia formed by the quad moved away from the meristem without the differentiation of floral organs (Supplemental Fig. S3B-E). In some cases, no new primordia were initiated, and in the absence of primordium formation, rings of undifferentiated cells developed similar to the ZND at the vegetative meristem (Supplemental Fig. S3B,F). In rare cases, organ formation at the inflorescence meristem ceased completely, resulting in a pin-like structure (7\% penetrance, $n=60$ ) (Supplemental Fig. S3F,G) similar to that seen in the pin1 auxin efflux carrier mutant (Okada et al. 1991). In addition to defects in organ positioning, flowers that did develop in the quad often had an abnormal structure, showing defects in floral organ number and positioning relative to wildtype plants (data not shown).

\section{The structure of the quad meristem is unaltered}

The disruption of primordium formation in the quad could be the indirect consequence of alterations in the structure of the meristem. To determine whether the cellular structure of the quad meristem differed from wild type, series of sections were made through meristems of both genotypes. In histological sections, the cellular structure of the apical dome of the quad meristem was unaltered relative to wild type (Supplemental Fig. S4).

To determine whether alterations in meristem activity were evident at the level of gene expression, we carried out in situ hybridization using gene-specific probes for the meristem marker genes CLAVATA3 (CLV3) and SHOOTMERISTEMLESS (STM). CLV3 has previously been characterized as a marker gene for the central zone of the meristem, and its expression is required to prevent proliferation of undifferentiated stem cells in the meristem center (Fletcher et al. 1999). In both wild-type and quad plants, $C L V 3$ expression was confined to the center of the meristem, with no sign of alterations in the strength or domain of expression in the quad, suggesting that the meristem itself was not enlarged (Fig. 3A-C).

Expression of the STM meristem maintenance gene has been previously characterized as occurring in both the central and peripheral zones of the meristem, with strong down-regulation in expression at the sites of developing primordia (Fig. 3D; Long et al. 1996; Long and Barton 2000). Similar expression of STM throughout the meristem was also seen in quad plants (Fig. 3E,F). STM expression was down-regulated in areas of the peripheral zone of quad plants in a manner similar to that seen in incipient primordia of wild-type plants (Fig. 3E,F, arrows). Down-regulation of STM expression was maintained in quad primordia that successfully grew out and also notably in abortive primordia, located some distance from the meristem (Fig. 3F, left-hand primordium). In the outermost regions of the $\mathrm{ZND}$, beyond the peripheral zone, STM expression was absent (Fig. 3E,F, arrowheads). Thus, cells entering the outer ZND lose STM expression, and therefore presumably meristematic activity, completely.

Expression of primordia specification genes is similar in wild-type and quad meristems

Defects in primordium initiation in the quad could result from the loss of expression of genes involved in primordium specification. To test whether this hypothesis could explain the lag phases in primordium initiation in the quad, we examined the expression of the AINTEGUMENTA $(A N T)$ and MONOPTEROS $(M P)$ genes.

$A N T$ is involved in the control of organ size and in wild-type plants shows expression in the peripheral zone of the meristem at the sites of primordium formation (Fig. 3G; Elliott et al. 1996; Long and Barton 1998, 2000; Mizukami and Fischer 2000). In quad plants, ANT expression was seen in a punctate fashion in the peripheral zone of the meristem (Fig. 3H,I, arrows). The patches of increased expression closest to the meristem could correspond to incipient primordia. The patches of expression further from the meristem are, however, likely to represent abortive primordia, suggesting that expression is maintained even as presumably abortive primordia move away from the meristem. This would indicate that even following specification, maintenance of ANT expression is insufficient to drive full primordium development and that additional factors are required.

The $m p$ mutant develops a pin-like inflorescence, which unlike pin 1 cannot initiate primordia in response to auxin application (Przemeck et al. 1996; Reinhardt et al. 2003). The MP gene, encoding an ARF-type auxin response transcription factor, is expressed at the sites of incipient primordia (Fig. 3J; Hardtke and Berleth 1998; Wenzel et al. 2007). This places $M P$ as a crucial factor in 
Bainbridge et al.

Figure 3. In situ analysis of expression of meristem and early primordium genes in wild type and the quadruple aux1 lax mutant. Central sections of a series of longitudinal sections through wild-type and quad vegetative meristems. (A) CLV3 in wild type. $(B, C) C L V 3$ in quad. $(D) S T M$ in wild type. $(E, F) S T M$ in quad. (G) $A N T$ in wild type. $(H, I) A N T$ in quad. (J) $M P$ in wild type. $(K, L) M P$ in quad. Black arrows indicate sites of STM down-regulation in incipient or bulging primordia. Black arrowheads indicate lack of STM expression in the outer ZND. Blue arrows indicate sites of $A N T$ up-regulation in incipient or bulging primordia. Blue arrowheads indicate lack of $A N T$ expression in the outer ZND. Gray arrows indicate $M P$ expression in the peripheral zone of the meristem. $(A, B, D, G, J)$ 33-39 DAG, $(C, E, F, H, I, K, L)$ 55-65 DAG. Bars, $50 \mu \mathrm{m}$.
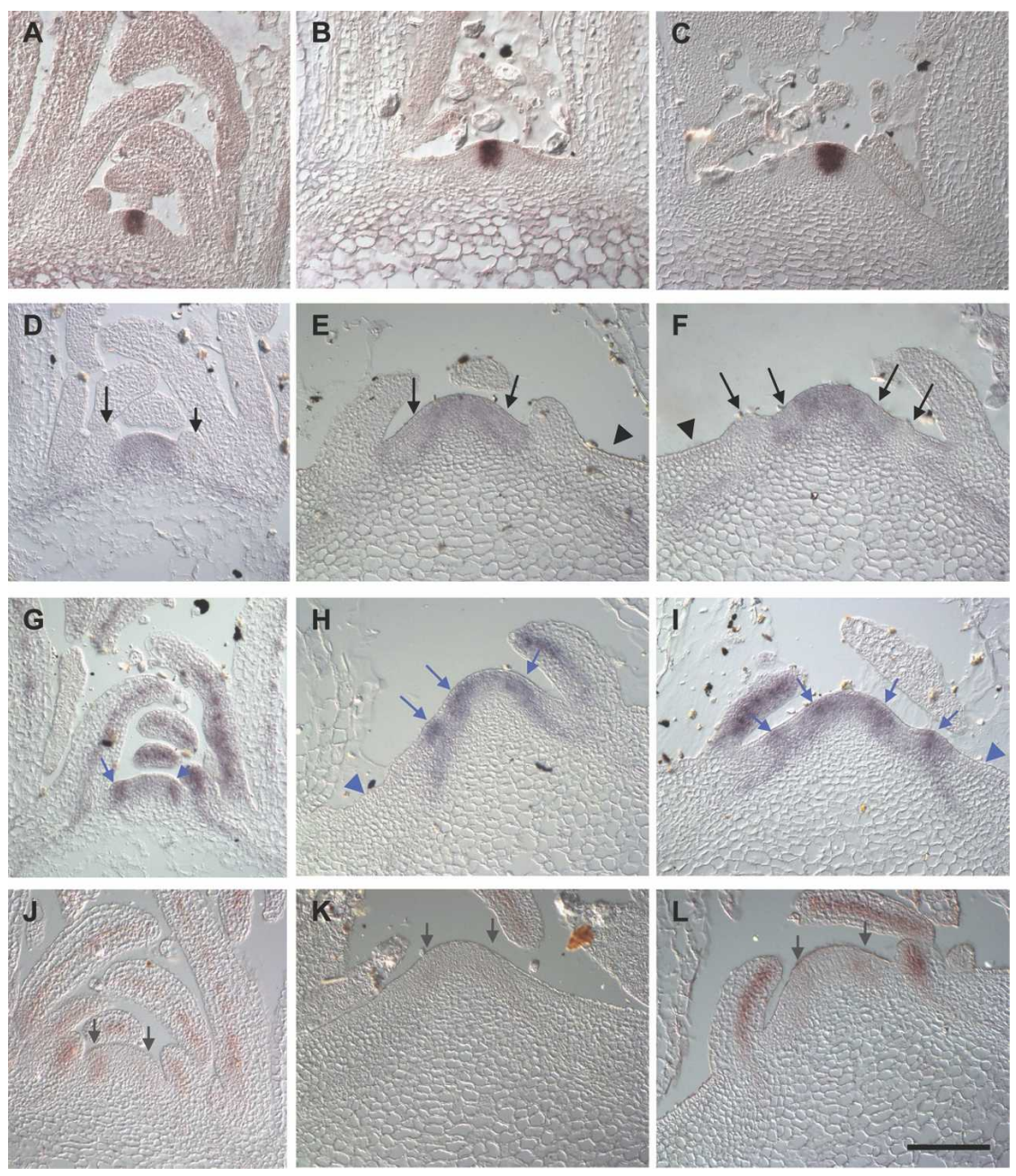

controlling the transcription of genes required for primordia outgrowth in response to the auxin signal. In the quad, MP expression was reduced in the peripheral zone of the meristem relative to that seen in wild-type plants (Fig. 3J-L). This could indicate that in cases where primordia fail to initiate or abort, MP expression is reduced, and this would be predicted to lead to reduced expression of genes required for primordium outgrowth. In outgrowing quad primordia, MP expression was seen at levels comparable with that in wild type (Fig. 3J-L).

\section{The AUX1 LAX genes act redundantly to maintain phyllotactic patterning}

Despite the disordered phyllotaxis first observed in the quadruple mutant, none of the aux1 lax single mutants exhibited defects in phyllotaxis (data not shown). In order to determine which of the AUX1 LAX genes were responsible for maintenance of regular phyllotaxis, we analyzed all double and triple mutant combinations.

In our analysis of all possible aux1 lax mutant combinations, four genotypes exhibited evidence of difficulties in primordium formation: the quad, aux1 lax1 lax2, aux1 lax1 lax3, and aux1 lax1 (Fig. 2; Supplemental Fig. S5). The common feature of all of these mutant combinations is the presence of mutations in both $A U X 1$ and $L A X 1$. At the inflorescence meristem, irregular phyllotaxis was again seen only in plants with these four mutant backgrounds (data not shown). This suggests a redundant function for $A U X 1$ and $L A X 1$ in maintaining regular phyllotactic patterning at both inflorescence and vegetative meristems. An increased severity in the phenotype of the quad and aux1 lax1 lax2 mutants was observed relative to aux1 lax1 and aux1 lax1 lax3 (Fig. 2; Supplemental Fig. S5). This suggests that the LAX2 gene could have a redundant function in maintaining regular phyllotaxis. The phenotypic data provide no evidence of function for LAX3 in organ initiation.

Functional redundancy between paralogous genes is most easily explained by overlapping expression patterns. In order to determine whether this could account for the functional redundancy of members of the $A U X 1$ $L A X$ family in maintaining phyllotactic patterning, we examined the expression patterns of these genes at the shoot apical meristem using promoter-GUS fusions. In the case of the $A U X 1$ and $L A X 3$, but not the $L A X 1$ and 
$L A X 2$ promoters, the functionality of the fragments used was confirmed by mutant complementation experiments.

Three of the AUX1 LAX genes showed expression in, or close to, the meristem. The two genes found to act functionally redundantly to maintain primordium initiation, $A U X 1$ and $L A X 1$, have partially overlapping, but distinct, expression patterns. In the vegetative meristem, overlapping expression occurs in the L1 of the peripheral zone and in the epidermis of developing primordia (Fig. 4A,B; Supplemental Figs. S6, S7A,B). Mutant phenotype analysis suggests that $L A X 2$ may also play a role in maintaining regular phyllotaxis, and while not expressed in the meristem itself, $L A X 2$ could fulfill this function through expression in the developing vasculature (Fig. $4 \mathrm{C}$; Supplemental Fig. S7C). One possibility is that LAX2 could increase the sink strength of developing primordia, pulling auxin out of the adjacent L1 layer and thus inhibiting primordium formation in this area. The lack of $L A X 3$ expression in the apical tissues (Fig. 4D; Supplemental Fig. S7D), the indistinguishable phyllotaxis phenotype of aux1 lax1 lax2 and the quad mutant, and the similar phenotypes of the aux1 lax1 and aux1 lax1 lax3 mutants suggest that $L A X 3$ is not involved in phyllotactic patterning.

Peaks in auxin signaling are altered in the aux1 lax1 lax2 triple mutant

The synthetic DR5::GFP auxin signaling reporter is frequently used as an indirect measure of auxin concentra-

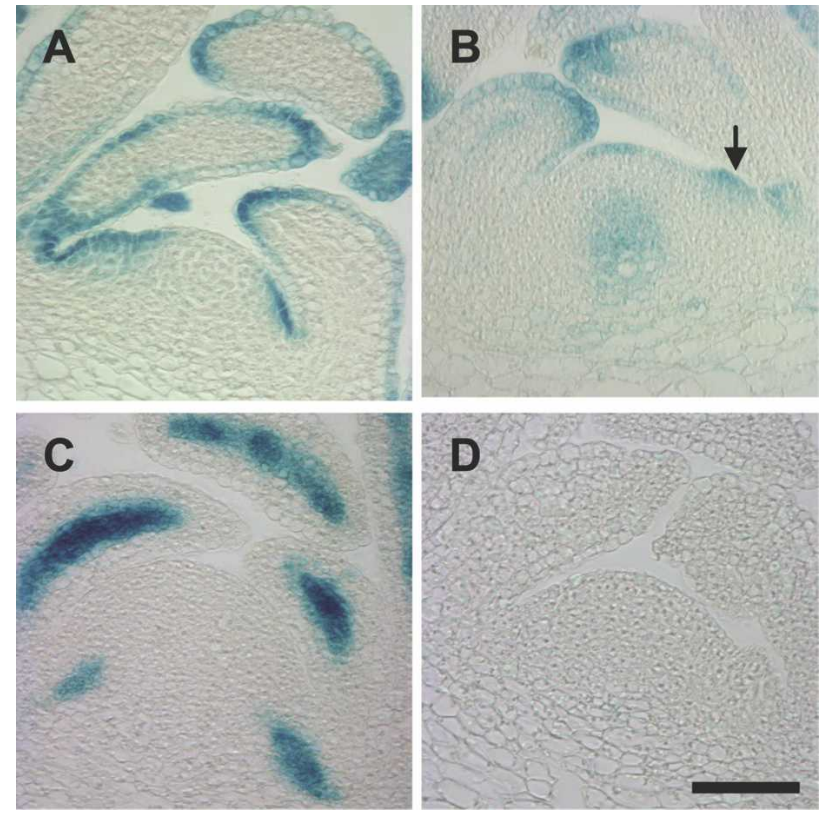

Figure 4. Expression of AUX1 and LAX promoter::GUS at vegetative meristems. Central, longitudinal sections through vegetative meristems in the wild-type background. (A) AUX1pr::GUS. (B) LAX1pr::GUS. Arrow indicates expression in the peripheral zone at the site of a bulging primordium. $(C)$ LAX2pr::GUS. (D) LAX3pr::GUS, 40 DAG. Histological GUS staining. Bars, $50 \mu \mathrm{m}$. tion at single-cell resolution. (Ulmasov et al. 1997; Sabatini et al. 1999; Casimiro et al. 2001; Benkova et al. 2003; Friml et al. 2003; Abas et al. 2006; de Reuille et al. 2006). To determine whether mutations in the $A U X 1$ and $L A X$ genes resulted in altered auxin distribution at the meristem, we examined DR5::GFP expression at inflorescence meristems of wild-type and aux1 lax mutant plants. In transverse images of wild-type meristems, the expression of DR5::GFP is clearly nonuniformly distributed in the L1 layer (Fig. 5A; Smith et al. 2006a). Peaks in $D R 5:: G F P$ signaling activity are arranged in a spiral pattern with the highest expression occurring at organ initiation sites, before visible bulging occurs, and in the youngest primordia (Fig. 5A; Smith et al. 2006a).

Analysis of DR5::GFP expression in aux1 or lax single mutant backgrounds revealed no alteration from the wild-type expression pattern, correlating with the lack of phyllotactic phenotype (data not shown). In the aux1 lax1 double mutant background, defects in the spacing and intensity of the peaks of DR5::GFP expression were apparent (data not shown). In the aux1 lax1 lax2 mutant background, the alteration in the DR5::GFP expression pattern was more striking than in aux1 lax1, reaffirming the contribution of $L A X 2$ (Fig. 5). As the phyllotaxis phenotype of aux1 lax1 lax2 was indistinguishable from the quad, all further DR5:: GFP expression analysis was carried out in this mutant background.

As described above, initiation and spacing of organs at the aux1 lax1 lax2 meristem is highly erratic, ranging from near spiral to a complete absence of organ initiation at any one time point. Consistent with the phyllotaxis phenotype, auxin signaling was dramatically altered in meristems of aux1 lax1 lax2 triple mutants. Peaks of auxin signaling were most often weaker and/or broader than in wild type $(n=43 / 74)$ (Fig. 5A,C,E). In addition, the arrangement of the peaks did not occur in the regular spiral of wild-type plants. In some cases, DR5::GFP was expressed relatively uniformly throughout the peripheral zone of the aux1 lax1 lax2 triple mutant $(n=25 / 74)$ (Fig. $5 \mathrm{G})$ or throughout the entire meristem, including the central zone $(n=4 / 74)$ (data not shown). These latter auxin distribution phenotypes were observed predominantly in meristems with strongly affected organogenesis.

In longitudinal sections through wild-type meristems, a striking feature of $D R 5:: G F P$ expression is that it is restricted to the L1 layer (Fig. 5I; Smith et al. 2006a). At sites where primordia initiate, expression extends into underlying cell layers, but, otherwise, GFP-positive cells were rarely seen outside the L1 layer (Fig. 5I). Longitudinal confocal scanning of aux1 lax1 lax2 meristems showed that DR5::GFP remained preferentially expressed in the L1 layer (Fig. 5K,M). In the majority of aux1 lax1 lax2 meristems, varying degrees of weak expression of DR5::GFP were also detectable in cells in inner layers (Fig. 5K,M). This could indicate reduced sequestering of auxin in the L1 layer.

In summary, aux1 lax1 lax2 meristems showed moderate alterations in the distribution of DR5::GFP signal between $\mathrm{L} 1$ and underlying layers. In contrast, major 

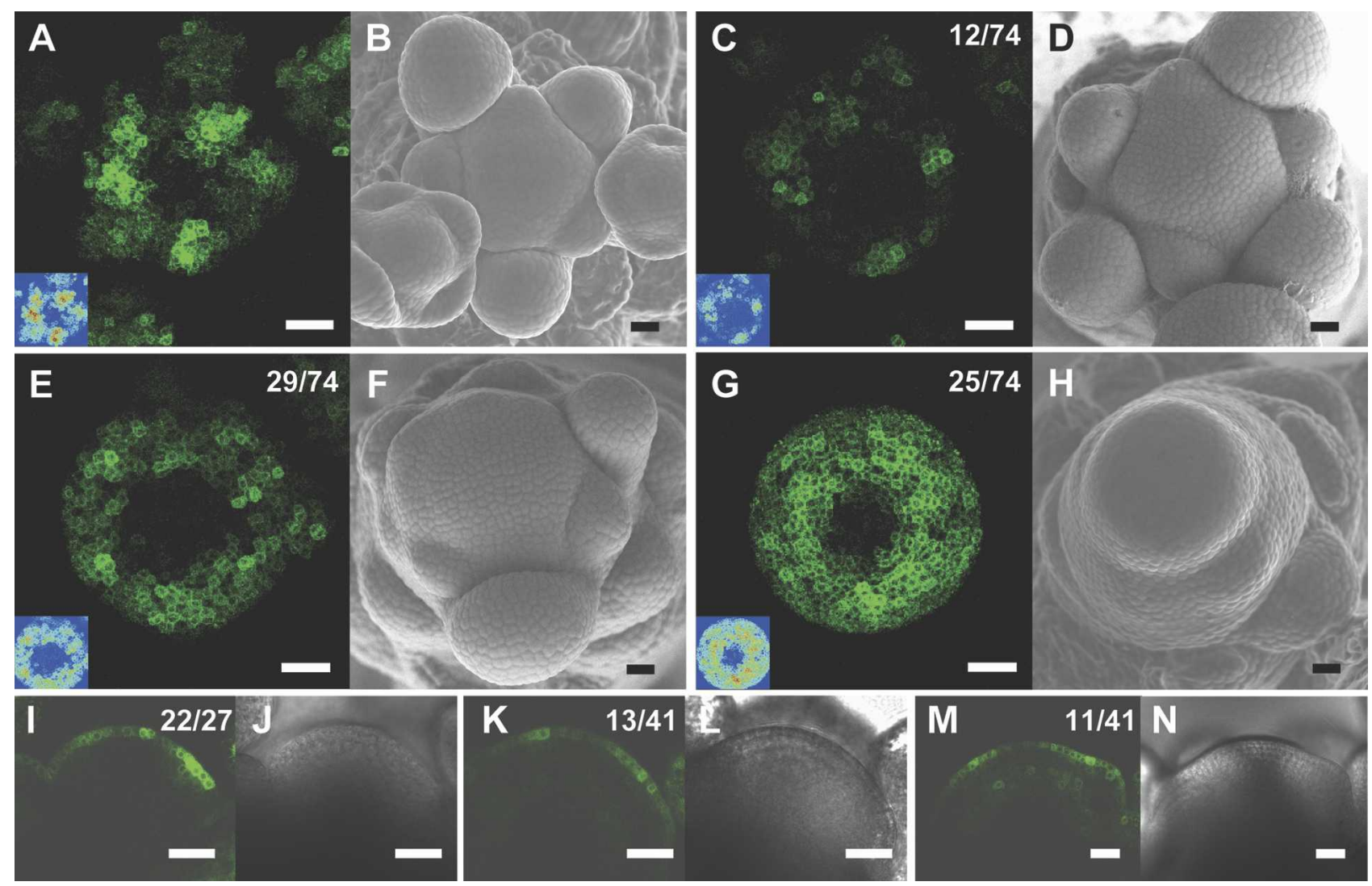

Figure 5. The pattern of $D R 5:: G F P$ expression is altered in aux1 lax1 lax2 triple mutant inflorescence meristems. (A) Maximal projection of transversal confocal scans of a wild-type meristem expressing DR5::GFP. (Inset) LUT signal intensity monitor. (Blue) Low intensity; (red) high intensity. (B) Scanning electron microscope image of the same wild-type meristem as in $A$. (C,E,G) Maximal projections of transversal confocal scans of DR5::GFP-expressing aux1 lax1 lax2 triple mutant meristems displaying increasingly severe phenotypes. (Inset) LUT signal intensity monitor. Representative meristems showing smaller and weaker peaks $(C)$, broader and less defined peaks $(E)$, or expression throughout the peripheral zone $(G) .(D, F, H)$ Scanning electron microscope images of the same meristems as in $C, E$, and $G$, respectively. (I) Longitudinal confocal section through a DR5::GFP-expressing wild-type meristem showing no GFP expression in inner layers, except below initiation sites. (J) Transmission light picture of the wild-type meristem shown in $I$. $(K, M)$ Longitudinal confocal section through two aux1 lax1 lax2 triple mutant meristems, showing very weak $(K)$ or weak (M) DR5 :: GFP expression in inner layers. $(L, N)$ Transmission light pictures of the meristems shown in $K$ and $M$. Bars, $25 \mu m$. Numbers indicate the number of occurrences over the total number of observations.

changes were seen in the distribution of DR5::GFP signal over the circumference of the meristem, with a strong correlation between the severity of the phenotype at the time of dissection and the loss of well-defined peaks of auxin signal. We hypothesize that this altered expression of DR5::GFP is likely to correspond to a loss of sharp, radial auxin gradients in the L1 of the peripheral zone in the aux1 lax1 lax2 mutant.

\section{The quad exhibits difficulties in coordinated} polarization of PIN1, but the basic mechanism of PIN1 polarization is not affected

To determine whether altered auxin gradients in aux1 lax mutant plants are accompanied by defects in PIN1 polarization, we carried out immunolocalization of the PIN1 protein at vegetative meristems of wild-type and quad plants. As previously reported in wild-type plants,
PIN1 was found to localize to the anticlinal membrane closest to the meristem summit, with convergence points at the sites of incipient primordia (Reinhardt et al. 2003). Sites of PIN1 convergent localization were clearly visible and arranged in a regular phyllotactic pattern around the wild-type meristem (Fig. 6A,B,E; Reinhardt et al. 2003). In inner layers, the punctate pattern of sites of convergent PIN1 localization around developing veins was clearly visible, with very low levels of signal detected outside of these regions (Fig. 6B,E).

In transversal sections through the quad meristem, PIN1 still showed evidence of polar localization in the L1 and L2 layers at the anticlinal membrane toward the meristem summit (Fig. 6C,D). Sites of possible convergent localization were visible, which could correspond to bulging or abortive primordia (Fig. 6C). Strikingly, however, the clear, punctate pattern of sites of PIN1 convergent localization seen in wild type was lost in the quad. 

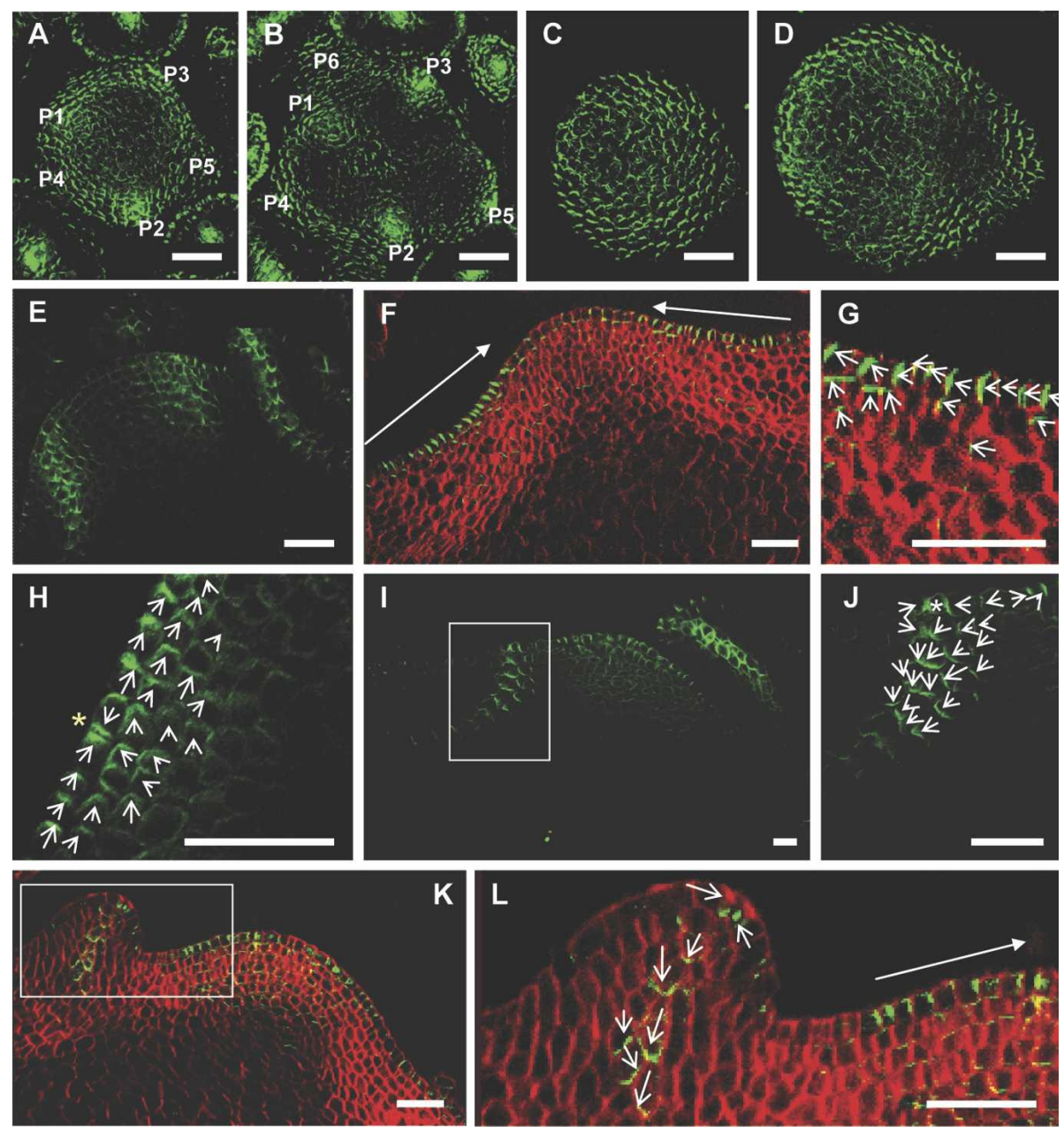

Figure 6. PIN1 polarization in the quadruple aux1 lax mutant. Green signal is PIN1 protein. Red signal is calcafluor staining of the cell walls. PIN1 localization visualized in maximal projections of serial optical sections, made by confocal imaging. $(A-D)$ Transversal sections. $(A, B)$ Wild-type meristem. Primordia labeled in order of initiation, with P1 the most recently initiated. $(A)$ Ten to twenty microns below the meristem summit, showing convergent PIN1 polarization in incipient and outgrowing primordia in the L1 of the peripheral zone. $(B)$ Twenty to thirty microns below the summit with PIN1 polarization toward developing vasculature of incipient and outgrowing primordia. $(C, D)$ quad meristem. $(C)$ Ten to twenty microns below the quad meristem summit, showing diffuse PIN1 polarization. $(D)$ Twenty to thirty microns below the summit, showing diffuse PIN1 polarization. $(E-L)$ Longitudinal sections. $(E)$ Central section through a wild-type meristem, showing convergent PIN1 localization in incipient and outgrowing primordia and basipetal PIN1 polarization along the sites of developing vasculature. $(F)$ Central section through a quad meristem with no recently initiated primordia, showing polarization of PIN1 toward the meristem summit in the L1 layer stretching to the periphery of the ZND. $(G)$ Enlargement of the right-hand ZND of the meristem shown in $F$, showing polarization of PIN1 toward the meristem summit in the $\mathrm{L} 1$ and apical polarization in the L2. $(H)$ Peripheral zone of a quad meristem with no newly initiated primordia showing convergent polarization in two cells of the PZ (yellow star). (I) A quad meristem with an incipient primordium in the PZ on the left and a recently initiated primordium to the right. $(J)$ Enlargement of the incipient primordium indicated by the rectangle in $I$, showing an L1 convergence point (white star) and basal polarization of PIN1 in underlying cell layers. $(K)$ A quad meristem with a newly initiated primordium in the PZ on the left. $(L)$ Enlargement of the newly initiated primordium indicated by the rectangle in $K$, showing basal polarization of PIN1. Closed-headed arrows show the general direction of polarization in a group of cells. Open-headed arrows show the direction of PIN1 polarization in each cell. White rectangles indicate areas enlarged in the next panel. Bars, $25 \mu \mathrm{m}$.

Regularly spaced sites of strongly convergent PIN1 polarization were not evident in the L1 or in underlying tissue layers of quad mutant meristems (Fig. 6C,D). Instead, a more diffuse pattern of PIN1 localization was seen, with an absence of both peaks and troughs in levels of expression.

To further characterize the direction of PIN1 polarization in the absence of primordium initiation, we also examined polarity in longitudinal sections through quad meristems. In meristems in which no new primordia had initiated, PIN1 showed clear polarization at the anticlinal membrane of L1 cells, closest to the meristem summit (Fig. 6F-I). This polarization at the anticlinal membrane stretched to the periphery of the ZND (Fig. 6F,G). Polar localization at the anticlinal membrane was also often seen in L2 cells, although in this cell layer apical 
polarity in the direction of the L1 layer was also seen (Fig. 6F-H). Convergent localization limited to pairs of adjacent cells was also occasionally seen in the peripheral zone (Fig. $6 \mathrm{H}$ ).

Longitudinal sections also showed that, in quad meristems, increased PIN1 expression often occurred in sub-L1 layers in the peripheral zone in the absence of primordium formation (Supplemental Fig. S8). These areas of increased expression in the peripheral zone often exhibited lateral polarization at the anticlinal membrane (Supplemental Fig. S8). In some cases, these sites of increased expression did not appear to form discrete sites of convergence, but stretched for some distance around the periphery (see convergence site 3 in Supplemental Fig. S8). In wild-type apices, convergence points were always limited to discrete sites in which strong, convergent polarization was seen (Supplemental Fig. S9).

The above evidence suggests that in the absence of primordium initiation, sites of clear and coordinated convergent PIN1 polarization do not occur in the quad. The quad mutant is, however, able to initiate primordia correctly, although this occurs at a reduced frequency relative to wild type. In the peripheral zone of quad meristems, clear PIN1 convergence points were found to occur, which presumably correspond to correctly initiating primordia (Fig. 6I,J). Although occurring at a lower frequency, sites of convergent PIN1 localization in the peripheral zone of the quad meristem were similar to those formed in wild type (Fig. 6E,I,J). As previously reported, basal PIN1 polarization occurred along the developing vasculature in bulging primordia in wild-type plants (Fig. 6E; Benkova et al. 2003; Reinhardt et al. 2003). Comparable basal polarization was seen in correctly initiating primordia in quad plants (Fig. 6I-L).

To summarize, these data suggest that two basic aspects of PIN1 polarization in the quad mutant are unaltered: formation of convergence points in the L1, and downward polarization in incipient and developing primordia. The frequency of PIN1 convergence point occurrence is, however, reduced, and the distribution of convergence points around the meristem is also altered.

\section{Discussion}

The rapid generation of dynamic auxin gradients at the shoot apical meristem is essential for regular primordium initiation and spacing (Benkova et al. 2003; Reinhardt et al. 2003; Heisler et al. 2005; Smith et al. 2006a). Previous studies have focused on the role of the PIN1 auxin efflux carrier. Here, we show that in addition to PIN1, the AUX1 LAX family of auxin influx carriers is essential for stabilizing phyllotactic patterning. This finding indicates the existence of a previously uncharacterized level of complexity in the regulation of auxin distribution in the shoot apical meristem. We propose that the central phyllotactic patterning mechanism consists of an interactive loop between auxin and its polar transporter, PIN1, and that the AUX1 LAX importers stabilize this PIN1/auxin pattern generator.

The characteristics of the mutant phenotype can be summarized as follows. First, the structure of the quad meristem is unaltered relative to wild type. Mutants described previously as exhibiting irregular phyllotaxis show alterations in meristem size or structure, and it has been suggested that meristem size could have a major influence on phyllotaxis (Leyser and Furner 1992; Giulini et al. 2004). The quad, however, does not show alterations in meristem structure or the expression of meristem maintenance genes. Therefore, the phyllotactic alterations are not a consequence of defects in the meristem, but reflect a direct defect in the patterning mechanism. Second, the quad loses the ability to control the divergence angles between successive primordia, resulting in a highly irregular phyllotactic pattern. The clustering of primordia is in violation of Hofmeister's Rule that primordia form as far away as possible from previously initiated primordia. Third, lag periods in primordium initiation occur, resulting in the formation of zones of no distinction around the meristem. In addition, abortive primordia are initiated that move away from the meristem without undergoing full differentiation. Fourth, DR5::GFP signal at the quad shoot apical meristem becomes diffuse and loses the regularly spaced peaks and troughs seen in wild-type meristems. Fifth, sites of PIN1 convergent localization become less frequent and irregularly spaced in the quad in the floral and later stages of vegetative growth. Sixth, initial events in primordia specification occur correctly whether the primordia grow out normally or abort. Together, these striking phenotypes of the quad mutant raise the question of how the AUX1 LAX family of auxin influx carriers assists in maintaining regular phyllotaxis and organ initiation at the shoot meristem.

\section{The role of AUX1 LAX influx carriers in maintaining} phyllotactic patterning

As described above, it has been previously suggested that AUX1 could enable regular phyllotactic patterning by retaining auxin within the L1 layer of the meristem (Reinhardt et al. 2003; Heisler et al. 2005). Here, we show that in addition to $A U X 1$, the $L A X 1$ gene also shows expression within the L1 layer of the meristem, suggesting that these two genes could act in concentrating auxin into the L1. In backgrounds where both AUX1 and LAX1 were mutated, defects in primordium initiation were evident. The levels of DR5::GFP expression in the L1 layer of these plants were, however, similar to those seen in wild type. In comparison, DR5::GFP levels in the L1 of the pin1 mutant are greatly reduced (Smith et al. 2006b). Together, these data suggest that the retention or concentration of auxin into the L1 layer of the shoot meristem may involve the PIN1 protein.

In the aux1 lax1 lax2 mutant, transversal images showed that both peaks and troughs in DR5::GFP expression were lost. This indicates that $A U X 1, L A X 1$, and $L A X 2$ are involved in controlling the circumferential distribution of auxin at the meristem. In addition, the distribution of PIN1 shows a similar diffuse pattern, with sites of clearly convergent localization occurring less of- 
ten than in wild-type plants and lacking regular spacing. This suggests that aux1 lax mutations influence the ability of the PIN1 protein to polarize effectively and to generate convergence points (Fig. 7). Thus, contrary to expectation, the importers appear to have little effect on the sequestration of auxin in the L1, but instead affect the redistribution of auxin within the $\mathrm{L} 1$.

\section{A model for the interaction of the PIN1 and AUX1 $L A X$ genes in controlling phyllotactic patterning}

The continuous generation of new primordia around the circumference of the meristem requires the rapid and dynamic formation of auxin peaks. Simulation models for auxin-mediated phyllotaxis propose that PIN1 orients toward a neighboring cell with a higher auxin concentration (Jonsson et al. 2006; Smith et al. 2006a). The underlying molecular mechanism is, however, still unknown. AUX1 LAX could be part of the mechanism that orients PIN1 toward cells with highest auxin concentration. The simplest hypothesis would be that a direct interaction between the two types of transporters is required for this "up-the-gradient polarization." The observation that PIN1 is able to polarize correctly in initiating and bulging primordia in the quad, however, argues against this hypothesis.

An alternative hypothesis for the loss of coordinated PIN1 polarization and of regularly spaced auxin peaks in the quad supposes that the aux1 lax mutations result in a general slowing in the rate of directional auxin transport (Fig. 7). This could be particularly relevant in the L1, where the rapid and dynamic generation of auxin peaks is required. This hypothesis predicts that in the quad backgrounds, PIN1 is still fully functional, and therefore effectively polarizes in response to the auxin concentration in neighboring cells (Fig. 7; Jonsson et al. 2006; Smith et al. 2006a). PIN1 can, however, only transport intracellular auxin. Once outside the cell, a reduced amount of auxin is able to enter the next cell in the absence of active auxin uptake. This increases random diffusion and reduces the efficiency of active, directional transport. With less coordinated PIN1 polarization and less efficient transport between cells, peaks and troughs in auxin concentration would occur less regularly and with less regular spacing. This would account for the clustering and irregular spacing of primordia in the quad mutant. A major challenge will be to incorporate the auxin importers into plausible quantitative models of phyllotaxis. Our preliminary attempts indicate that this will require substantial advances in our understanding of the molecular mechanisms of auxin transport, as well as advances in computer modeling, in order to generate a dynamic simulation of phyllotaxis including influx carriers and extracellular space.

An interesting feature of the primordia initiated by the quad mutant is that they show no apparent increase in size relative to those initiated by wild-type plants. This is in contrast to the pin 1 mutant, in which leaf primordia fusions frequently occur (Aida et al. 2002; Furutani et al. 2004). The absence of fusions in the quad is surprising

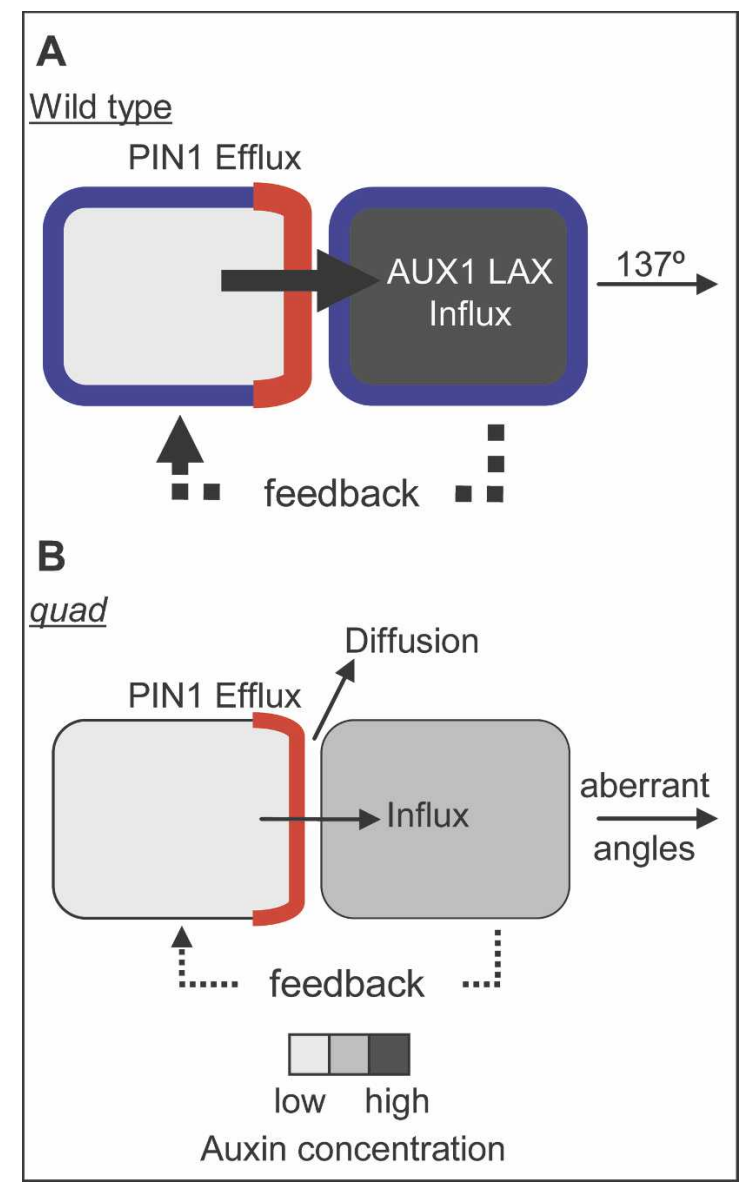

Figure 7. Model for the role of AUX1 LAX importers in phyllotaxis. (A) Two wild-type cells. (B) Two aux1 lax mutant cells. PIN1 (red), AUX1 LAX (blue), and auxin concentration (gray) are represented. In both wild-type and quad, PIN1 polarizes toward the neighboring cell with the higher auxin concentration and exports auxin from the cell. $(A)$ In wild type, auxin is rapidly taken up into the next cell due to AUX1 LAX function, causing an increase in auxin concentration in this cell. The higher auxin concentration in this cell feeds back to control PIN1 polarity. This causes further efflux toward this cell, which is rapidly taken up and further increases auxin concentration. This tightly controlled generation of peaks and troughs in auxin concentration generates a regular angle of $137^{\circ}$ between successive primordia. $(B)$ In aux1 lax mutants, auxin leaves the cell via PIN1mediated efflux. The absence of AUX1 LAX reduces the efficiency with which auxin is taken up into the next cell, and therefore more diffusion of auxin can occur in the apoplastic space. Some auxin may enter the next cell to increase the auxin concentration of the cell. Any increase in auxin concentration would feed back to control PIN1 polarity, increasing efflux and further increasing auxin concentration in the next cell. This less regulated generation of auxin peaks results in irregular spacing between primordia. In addition, peaks above the threshold for primordium initiation, but below the threshold level for full primordium differentiation, would result in the abortive primordia seen in the quad.

considering the initiation of primordia in very close proximity to each other and the broad maxima of DR5::GFP expression. The lack of fusion in the quad 
implies that following primordium initiation, an inhibitory field is rapidly formed, prohibiting initiation of another primordium in the immediate vicinity. This inhibitory field must be set up very early in the process of primordium initiation, as even abortive primordia do not fuse. This boundary function is likely to require the PIN1 protein, which has been shown to be vital in the establishment of organ boundaries (Aida et al. 2002; Furutani et al. 2004). Thus, in contrast to a previous study performed with the import inhibitor 1-napthoxyactetic acid (NOA), we now propose that the importers are not involved in restricting primordium width (Stieger et al. 2002). However, they may still influence the action of PIN1 in this respect (Reinhardt et al. 2003).

In wild-type plants, STM, $M P$, and $A N T$ are expressed in a regular phyllotactic pattern. In the pin 1 mutant, they are expressed ubiquitously in the peripheral zone of the meristem, showing that PIN1 is at the core of the phyllotactic patterning of these genes (Vernoux et al. 2000). In the quad, however, these three genes retain many elements of their phyllotactic information, but the spacing has become irregular. This supports the notion that the PIN-based patterning mechanism is essentially still functioning but unstable. The occurrence of aborted primordia can be explained by the generation of weaker peaks of auxin that are sufficient for the early stages of primordium initiation, but insufficient to trigger later events. The notion that weaker peaks of auxin may result in reduced expression of genes necessary for primordium initiation and development is supported by the reduced levels of $M P$ expression in the quad background. The expression of the $M P$ auxin signaling transcription factor is essential for primordium initiation as evidenced by the naked inflorescence meristem of the $m p$ mutant (Przemeck et al. 1996; Reinhardt et al. 2003). It remains to be determined whether $M P$ expression is responding directly to auxin peaks or to downstream signaling events necessary for primordium initiation.

The role of the AUX1 LAX family in stabilizing phyllotaxis against differential environmental or developmental signals

The phenotype of the quad suggests that the AUX1 LAX genes are of particular importance at certain stages of development and/or under particular environmental conditions. At the inflorescence meristem, the quad mutant shows irregular phyllotaxis under both long- and short-day conditions. At the vegetative meristem, however, the quad shows no defects in phyllotactic patterning under long-day conditions. When grown in a short photoperiod, where the vegetative period is longer, regular spiral phyllotaxis is maintained for the first month of growth, with defects first evident from 35 DAG.

One explanation for the differences in severity of the phyllotaxis phenotype could be redundancy of AUX1 LAX function with other transporters expressed during early stages of vegetative growth. In addition to the AUX1 LAX family, an involvement for the PGP4 protein in auxin import has also been suggested, although ex- pression at the shoot meristem has not been characterized (Santelia et al. 2005; Terasaka et al. 2005a,b). An alternative hypothesis is that auxin levels, transport, or signaling response may differ under different light conditions, and this could be critical for plants in which import is impaired. The involvement of light-regulated auxin control of leaf primordia growth has been demonstrated recently and, increasingly, links between light and auxin pathways have been found (Heggie and Halliday 2005; Carabelli et al. 2007). A final hypothesis for the differences in the strength of phenotype could be the gradual increase in meristem size seen in wild-type plants during vegetative development. As the meristem becomes bigger, the importance of rapid auxin uptake in maintaining gradients in auxin concentration could become crucial. At this point, the quad mutant could start to experience difficulties in maintaining auxin gradients, whereas AUX1 LAX function in wild-type plants would help to generate peaks of auxin and stabilize phyllotaxis.

In conclusion, our findings are consistent with previous studies suggesting that the PIN1 auxin efflux carrier is the key component in coordinating the polar auxin transport necessary for the generation of regular phyllotactic patterns. Our study of the aux1 lax quadruple mutant, however, indicates that AUX1 LAX-mediated uptake is critical in stabilizing auxin gradients and assisting in coordinated PIN1 polarization at particular stages in development or under challenging environmental conditions. The tissue-specific expression of the AUX1 $L A X$ genes is therefore likely to provide the plant with an additional point of regulation. The role of the $A U X 1$ $L A X$ genes in these processes and the mechanism by which developmental and environmental stimuli could feed into this system will be an important field for future study.

\section{Materials and methods}

\section{Plant material and growth conditions}

All Arabidopsis thaliana lines were in the Columbia background. Plants were grown on soil under a short photoperiod (8 h light, $60 \%$ humidity, $20^{\circ} \mathrm{C}-22^{\circ} \mathrm{C}$ day temperature, irradiance $100 \mu \mathrm{Em}^{-2 \mathrm{~s}-2}$ ), except for the plants shown in Figure 1, A and $\mathrm{B}$, which were grown under a long photoperiod $(16 \mathrm{~h}$ light, irradiance $150 \mu \mathrm{Em}^{-2 \mathrm{~s}-2}$ ).

dSpm insertion lines for the Arabidopsis LAX1 (At5g01240), $L A X 2$ (At2g21050), and LAX3 (At1g77690) genes were identified in the SLAT population 1 using a two-step screening approach as described (Swarup et al. 2008). Genomic DNA was initially isolated from pools of $50 \mathrm{dSpm}$ lines, then genomic sequences flanking dSpm elements were PCR-amplified, spotted onto a nitrocellulose filter, and hybridized with LAX gene-specific probes. Genomic DNA was isolated from individual plants within a positively hybridizing pool, which was screened using pairs of dSpm and LAX PCR primers, to identify dSpm insertion lines within each LAX gene sequence. LAX1 (At5G01240) contains an insertion in the fourth exon, 945 base pairs (bp) downstream from the ATG. LAX2 (At2G21050) contains an insertion in the second intron $452 \mathrm{bp}$ downstream from the ATG, and RT-PCR showed an absence of mRNA. LAX3 (At1G77690) con- 
tains an insertion at the beginning of the sixth exon, $1316 \mathrm{bp}$ downstream from the ATG (Swarup et al. 2008).

The aux1-21 mutant was generated by X-ray mutagenesis and has been described previously (Marchant and Bennett 1998). The roots of the aux1-21 mutant are resistant to the synthetic auxin 2,4-D (Marchant and Bennett 1998), whereas none of the lax mutants exhibit this response. In the generation of multiple mutant and transgenic lines, aux1-21 was therefore selected by 2,4-D resistance. In generating multiple mutant combinations, the lax mutants were selected by PCR for the absence of a wild-type band using the following gene-specific primers: $L A X 1$, 5'-ATATGGTTGCAGGTGGCACA-3' and 5'-GTAACCGGC AAAAGCTGCA- ${ }^{\prime}$; LAX2, $5^{\prime}$-ATGGAGAACGGTGAGAAA GCAGC-3' and 5'-CGCAGAAGGCAGCGTTAGCG-3'; LAX3, 5'-TACTTCACCGGAGCCACCA- ${ }^{\prime}$ and $5^{\prime}$-TGATTGGTCC GAAAAAGG-3'. In lines negative for the wild-type band, the presence of the T-DNA insertion was verified using the forward primers listed above along with the reverse primer $5^{\prime}$-AAG CACGACGGCTGTAGAATAG-3', which primes from the transposon insertion.

DR5::GFP refers to the DR5rev::GFP line (Benkova et al. 2003). Crosses of DR5::GFP wild type were made with the quad. In the F2 generation, segregating plants were selected on the basis of the narrow leaf phenotype characteristic of aux1 lax1 lax2 and quad mutants and DR5:: GFP-positive root tips. Homozygous DR5::GFP-expressing aux1 lax1 lax2 plants were confirmed in the F4 generation by 2,4-D resistance and $L A X$ gene-specific PCR as described above.

AUX1pr::AUX1:HA- and AUX1pr::GUS-expressing plants have been described previously (Marchant et al. 1999; Swarup et al. 2005). To generate LAX1, LAX2, and LAX3pr::GUS constructs, upstream sequences of $2.8 \mathrm{~kb}, 2 \mathrm{~kb}$, and $1.8 \mathrm{~kb}$, respectively, were PCR-amplified and fused upstream to the uidA coding sequence in a Bin19-based binary vector. Transformation of Agrobacterium (C58) and Arabidopsis was carried out as described (Swarup et al. 2001).

\section{Macroscopic analysis of leaf initiation and measurement} of angles

Photographs were taken of plants every $2-3 \mathrm{~d}$ from germination until flowering. Leaves were numbered in order of initiation, and the number of new leaves was recorded. The angles measured at $48,57,69$, and 81 DAG were between the two smallest, consecutively formed leaves that were macroscopically measurable with accuracy. The smallest was defined as being $\geq 9 \mathrm{~mm}$ in length.

\section{In situ detections of $m R N A$, proteins, and GUS expression}

In situ hybridizations used digoxigenin-labeled riboprobes as described previously (Vernoux et al. 2000). The STM, ANT, and $C L V 3$ probes were gifts from Dr. Jan Traas (INRA, Lyon, France; Vernoux et al. 2000). The $M P$ probe was a gift from Dr. Thomas Berleth (University of Toronto, Canada; Hardtke and Berleth 1998). To ensure the size of the meristem was unaltered in quad plants, CLV3 in situs were first carried out on wild-type and quad tissue fixed at 36 DAG. Further in situs used wild-type tissue fixed at 33-39 DAG to ensure that all plants analyzed had not undergone floral transition. quad tissue was fixed at 55-65 DAG, thus ensuring the majority of plants had arrested primordium formation and ZND formation.

Protein detections, fixations, and immunofluorescent detection of PIN1 and AUX1-HA were carried out as described (Swarup et al. 2001; Friml et al. 2002; Reinhardt et al. 2003). The blocking buffer used was 5\% BSA (Sigma), 5\% normal goat se- rum (Aurion), and $0.1 \%$ cold-water fish skin gelatin (Aurion) in phosphate-buffered saline. The primary antibody was diluted in $0.1 \%$ acetylated BSA (Aurion). Primary antibodies against PIN1 and AUX1:HA were as described previously (Reinhardt et al. 2003). Alexa Fluor 488-conjugated goat secondary antibodies were used ( 1 in 300; Molecular Probes). PIN1 slides were counterstained with $10 \mu \mathrm{g} / \mathrm{mL}$ calcofluor and mounted as described (Reinhardt et al. 2003).

AUX1 and LAXpr::GUS plants were prefixed, GUS-stained, fixed, and embedded as described previously (Sessions et al. 1999). Samples were incubated in GUS stain containing $10 \mathrm{mM}$ potassium ferrocyanide and $10 \mathrm{mM}$ potassium ferricyanide as described (Sessions et al. 1999) for $14-16$ h at $37^{\circ} \mathrm{C}$.

\section{Plastic-embedded tissue sections}

Plastic sections were prepared as described previously (Loreto et al. 2001) with the modification that $\mathrm{OsO}_{4}$ was omitted.

\section{Microscopy}

In situ hybridization and GUS-stained and histological sections were viewed under a Zeiss Axioskop 2 equipped with an Axiocam camera.

Confocal analysis of immunolocalizations was carried out using a Leica inverted confocal laser-scanning microscope (DMIRE2) with a water immersion $20 \times$ or $63 \times$ objective. Excitation was at $488 \mathrm{~nm}$ at $16 \%$ of full power and collection between 505 and $545 \mathrm{~nm}$. For detection of calcofluor signal, excitation with a 405 laser at $19 \%$ and collection between 420 and $470 \mathrm{~nm}$ was used. For DR5::GFP expression analysis, a Leica upright confocal laser-scanning microscope (DMRXE7) with a long-working-distance water immersion objective $(\times \mathrm{L} 63 / 0.90$ U-V-I) was used. Excitation was at $488 \mathrm{~nm}$ with $20 \%$ power. The collection wavelengths were $500-550 \mathrm{~nm}$ for GFP.

For scanning electron microscopic analysis, freshly dissected tissue was viewed with a S-3500N variable pressure scanning electron microscope (Hitachi) equipped with a cool stage.

\section{Acknowledgments}

We thank Nick James for his involvement in the generation of the quadruple mutant; Kamal Swarup for RT-PCR analysis of the lax mutants; Ueli Klahre, Naomi Nakayama, Richard Smith, and Saiko Yoshida for helpful discussions and comments on the manuscript; and Christopher Ball, Rebecca Alder, and Tabea Büschlen for expert plant care. This work was funded by the EU "MechPlant" project and the Swiss NSF.

\section{References}

Abas, L., Benjamins, R., Malenica, N., Paciorek, T., Wirniewska, J., Moulinier-Anzola, J.C., Sieberer, T., Friml, J., and Luschnig, C. 2006. Intracellular trafficking and proteolysis of the Arabidopsis auxin-efflux facilitator PIN2 are involved in root gravitropism. Nat. Cell Biol. 8: 249-256.

Aida, M., Vernoux, T., Furutani, M., Traas, J., and Tasaka, M. 2002. Roles of PIN-FORMED1 and MONOPTEROS in pattern formation of the apical region of the Arabidopsis embryo. Development 129: 3965-3974.

Benkova, E., Michniewicz, M., Sauer, M., Teichmann, T., Seifertova, D., Jurgens, G., and Friml, J. 2003. Local, effluxdependent auxin gradients as a common module for plant organ formation. Cell 115: 591-602.

Bennett, M.J., Marchant, A., Green, H.G., May, S.T., Ward, S.P., 
Millner, P.A., Walker, A.R., Schulz, B., and Feldmann, K.A. 1996. Arabidopsis AUX1 gene: A permease-like regulator of root gravitropism. Science 273: 948-950.

Carabelli, M., Possenti, M., Sessa, G., Ciolfi, A., Sassi, M., Morelli, G., and Ruberti, I. 2007. Canopy shade causes a rapid and transient arrest in leaf development through auxin-induced cytokinin oxidase activity. Genes \& Dev. 21: 18631868.

Casimiro, I., Marchant, A., Bhalerao, R.P., Beeckman, T., Dhooge, S., Swarup, R., Graham, N., Inze, D., Sandberg, G., Casero, P.J., et al. 2001. Auxin transport promotes Arabidopsis lateral root initiation. Plant Cell 13: 843-852.

de Reuille, P.B., Bohn-Courseau, I., Ljung, K., Morin, H., Carraro, N., Godin, C., and Traas, J. 2006. Computer simulations reveal properties of the cell-cell signaling network at the shoot apex in Arabidopsis. Proc. Natl. Acad. Sci. 103: $1627-1632$.

De Smet, I., Tetsumura, T., De Rybel, B., Frey, N.F.D., Laplaze, L., Casimiro, I., Swarup, R., Naudts, M., Vanneste, S., Audenaert, D., et al. 2007. Auxin-dependent regulation of lateral root positioning in the basal meristem of Arabidopsis. Development 134: 681-690.

Elliott, R.C., Betzner, A.S., Huttner, E., Oakes, M.P., Tucker, W.Q.J., Gerentes, D., Perez, P., and Smyth, D.R. 1996. AINTEGUMENTA, an APETALA2-like gene of Arabidopsis with pleiotropic roles in ovule development and floral organ growth. Plant Cell 8: 155-168.

Fletcher, L.C., Brand, U., Running, M.P., Simon, R., and Meyerowitz, E.M. 1999. Signaling of cell fate decisions by CLAVATA3 in Arabidopsis shoot meristems. Science 283: 19111914.

Friml, J., Benkova, E., Blilou, I., Wisniewska, J., Hamann, T., Ljung, K., Woody, S., Sandberg, G., Scheres, B., Jurgens, G., et al. 2002. AtPIN4 mediates sink-driven auxin gradients and root patterning in Arabidopsis. Cell 108: 661-673.

Friml, J., Vieten, A., Sauer, M., Weijers, D., Schwarz, H., Hamann, T., Offringa, R., and Jurgens, G. 2003. Efflux-dependent auxin gradients establish the apical-basal axis of Arabidopsis. Nature 426: 147-153.

Furutani, M., Vernoux, T., Traas, J., Kato, T., Tasaka, M., and Aida, M. 2004. PIN-FORMED1 and PINOID regulate boundary formation and cotyledon development in Arabidopsis embryogenesis. Development 131: 5021-5030.

Giulini, A., Wang, J., and Jackson, D. 2004. Control of phyllotaxy by the cytokinin-inducible response regulator homologue ABPHYL1. Nature 430: 1031-1034.

Hardtke, C.S. and Berleth, T. 1998. The Arabidopsis gene MONOPTEROS encodes a transcription factor mediating embryo axis formation and vascular development. EMBO $I$. 17: 1405-1411.

Heggie, L. and Halliday, K.J. 2005. The highs and lows of plant life: Temperature and light interactions in development. Int. J. Dev. Biol. 49: 675-687.

Heisler, M.G. and Jonsson, H. 2006. Modeling auxin transport and plant development. J. Plant Growth Regul. 25: 302-312.

Heisler, M.G., Ohno, C., Das, P., Sieber, P., Reddy, G.V., Long, J.A., and Meyerowitz, E.M. 2005. Patterns of auxin transport and gene expression during primordium development revealed by live imaging of the Arabidopsis inflorescence meristem. Curr. Biol. 15: 1899-1911.

Hofmeister, W. 1868. Allgemeine Morphologie der Gewächse. In Handbuch der physiologischen Botanik; Band 1, Abteilung 2, pp. 405-664. W. Engelmann, Leipzig.

Jonsson, H., Heisler, M.G., Shapiro, B.E., Meyerowitz, E.M., and Mjolsness, E. 2006. An auxin-driven polarized transport model for phyllotaxis. Proc. Natl. Acad. Sci. 103: 1633-1638.
Kramer, E.M. 2004. PIN and AUX/LAX proteins: Their role in auxin accumulation. Trends Plant Sci. 9: 578-582.

Kramer, E.M. 2006. How far can a molecule of weak acid travel in the apoplast or xylem? Plant Physiol. 141: 1233-1236.

Kramer, E.M. and Bennett, M.J. 2006. Auxin transport: A field in flux. Trends Plant Sci. 11: 382-386.

Kuhlemeier, C. 2007. Phyllotaxis. Trends Plant Sci. 12: 143150.

Leyser, H.M.O. and Furner, I.J. 1992. Characterization of 3 shoot apical meristem mutants of Arabidopsis thaliana. Development 116: 397-403.

Long, J.A. and Barton, M.K. 1998. The development of apical embryonic pattern in Arabidopsis. Development 125: 30273035.

Long, J. and Barton, M.K. 2000. Initiation of axillary and floral meristems in Arabidopsis. Dev. Biol. 218: 341-353.

Long, J.A., Moan, E.I., Medford, J.I., and Barton, M.K. 1996. A member of the KNOTTED class of homeodomain proteins encoded by the STM gene of Arabidopsis. Nature 379: 6669.

Loreto, F., Mannozzi, M., Maris, C., Nascetti, P., Ferranti, F., and Pasqualini, S. 2001. Ozone quenching properties of isoprene and its antioxidant role in leaves. Plant Physiol. 126: 993-1000.

Marchant, A. and Bennett, M.J. 1998. The Arabidopsis AUX1 gene: A model system to study mRNA processing in plants. Plant Mol. Biol. 36: 463-471.

Marchant, A., Kargul, J., May, S.T., Muller, P., Delbarre, A., Perrot-Rechenmann, C., and Bennett, M.J. 1999. AUX1 regulates root gravitropism in Arabidopsis by facilitating auxin uptake within root apical tissues. EMBO J. 18: 2066-2073.

Marchant, A., Bhalerao, R., Casimiro, I., Eklof, J., Casero, P.J., Bennett, M., and Sandberg, G. 2002. AUX1 promotes lateral root formation by facilitating indole-3-acetic acid distribution between sink and source tissues in the Arabidopsis seedling. Plant Cell 14: 589-597.

Mizukami, Y. and Fischer, R.L. 2000. Plant organ size control: AINTEGUMENTA regulates growth and cell numbers during organogenesis. Proc. Natl. Acad. Sci. 97: 942-947.

Okada, K., Ueda, J., Komaki, M.K., Bell, C.J., and Shimura, Y. 1991. Requirement of the auxin polar transport system in early stages of Arabidopsis floral bud formation. Plant Cell 3: 677-684.

Parry, G., Marchant, A., May, S., Swarup, R., Swarup, K., James, N., Graham, N., Allen, T., Martucci, T., Yemm, A., et al. 2001. Quick on the uptake: Characterization of a family of plant auxin influx carriers. J. Plant Growth Regul. 20: 217225.

Przemeck, G.K.H., Mattsson, J., Hardtke, C.S., Sung, Z.R., and Berleth, T. 1996. Studies on the role of the Arabidopsis gene MONOPTEROS in vascular development and plant cell axialization. Planta 200: 229-237.

Reinhardt, D., Mandel, T., and Kuhlemeier, C. 2000. Auxin regulates the initiation and radial position of plant lateral organs. Plant Cell 12: 507-518.

Reinhardt, D., Pesce, E.R., Stieger, P., Mandel, T., Baltensperger, K., Bennett, M., Traas, J., Friml, J., and Kuhlemeier, C. 2003. Regulation of phyllotaxis by polar auxin transport. Nature 426: $255-260$

Sabatini, S., Beis, D., Wolkenfelt, H., Murfett, J., Guilfoyle, T., Malamy, J., Benfey, P., Leyser, O., Bechtold, N., Weisbeek, P., et al. 1999. An auxin-dependent distal organizer of pattern and polarity in the Arabidopsis root. Cell 99: 463-472.

Santelia, D., Vincenzetti, V., Azzarello, E., Bovet, L., Fukao, Y., Duchtig, P., Mancuso, S., Martinoia, E., and Geisler, M. 2005. MDR-like ABC transporter AtPGP4 is involved in 
auxin-mediated lateral root and root hair development. FEBS Lett. 579: 5399-5406.

Sessions, A., Weigel, D., and Yanofsky, M.F. 1999. The Arabidopsis thaliana MERISTEM LAYER 1 promoter specifies epidermal expression in meristems and young primordia. Plant J. 20: 259-263.

Smith, R.S., Guyomarc'h, S., Mandel, T., Reinhardt, D., Kuhlemeier, C., and Prusinkiewicz, P. 2006a. A plausible model of phyllotaxis. Proc. Natl. Acad. Sci. 103: 1301-1306.

Smith, R.S., Kuhlemeier, C., and Prusinkiewicz, P. 2006b. Inhibition fields for phyllotactic pattern formation: A simulation study. Can. J. Bot. 84: 1635-1649.

Stieger, P.A., Reinhardt, D., and Kuhlemeier, C. 2002. The auxin influx carrier is essential for correct leaf positioning. Plant J. 32: 509-517.

Swarup, R., Friml, J., Marchant, A., Ljung, K., Sandberg, G., Palme, K., and Bennett, M. 2001. Localization of the auxin permease AUX1 suggests two functionally distinct hormone transport pathways operate in the Arabidopsis root apex. Genes \& Dev. 15: 2648-2653.

Swarup, R., Kargul, J., Marchant, A., Zadik, D., Rahman, A., Mills, R., Yemm, A., May, S., Williams, L., Millner, P., et al. 2004. Structure-function analysis of the presumptive Arabidopsis auxin permease AUX1. Plant Cell 16: 3069-3083.

Swarup, R., Kramer, E.M., Perry, P., Knox, K., Leyser, H.M.O., Haseloff, J., Beemster, G.T.S., Bhalerao, R., and Bennett, M.J. 2005. Root gravitropism requires lateral root cap and epidermal cells for transport and response to a mobile auxin signal. Nat. Cell Biol. 7: 1057-1065.

Swarup, K., Benkova, E., Swarup, R., Casimiro, I., Péret, B., Yang, Y., Parry, G., Nielsen, E., De Smet, I., Vanneste, S., et al. 2008. The auxin influx carrier LAX3 promotes lateral root emergence. Nat. Cell Biol. (in press).

Terasaka, K., Blakeslee, J.J., Titapiwatanakun, B., Peer, W.A., Bandyopadhyay, A., Makam, S.N., Lee, O.R., Richards, E.L., Murphy, A.S., Sato, F., et al. 2005a. PGP4, an ATP binding cassette P-glycoprotein, catalyzes auxin transport in Arabidopsis thaliana roots. Plant Cell 17: 2922-2939.

Terasaka, K., Murphy, A.S., Sato, F., and Yazaki, K. 2005b. Gene expression and functional analyses of a P-glycoprotein-like (PGP) ABC protein gene, AtPGP4, in Arabidopsis thaliana. Plant Cell Physiol. 46: S35.

Ulmasov, T., Murfett, J., Hagen, G., and Guilfoyle, T.J. 1997. Aux/IAA proteins repress expression of reporter genes containing natural and highly active synthetic auxin response elements. Plant Cell 9: 1963-1971.

Vernoux, T., Kronenberger, J., Grandjean, O., Laufs, P., and Traas, J. 2000. PIN-FORMED 1 regulates cell fate at the periphery of the shoot apical meristem. Development 127: 5157-5165.

Wenzel, C.L., Schuetz, M., Yu, Q., and Mattsson, J. 2007. Dynamics of MONOPTEROS and PIN-FORMED1 expression during leaf vein pattern formation in Arabidopsis thaliana. Plant J. 49: 387-398.

Yang, Y.D., Hammes, U.Z., Taylor, C.G., Schachtman, D.P., and Nielsen, E. 2006. High-affinity auxin transport by the AUX1 influx carrier protein. Curr. Biol. 16: 1123-1127. 


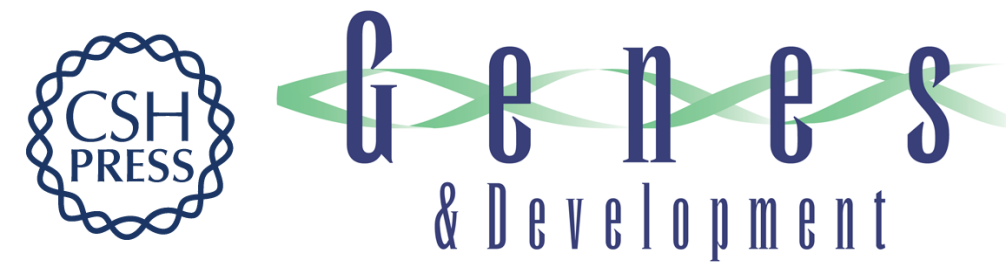

\section{Auxin influx carriers stabilize phyllotactic patterning}

Katherine Bainbridge, Soazig Guyomarc'h, Emmanuelle Bayer, et al.

Genes Dev. 2008, 22:

Access the most recent version at doi:10.1101/gad.462608

Supplemental

Material

References This article cites 52 articles, 26 of which can be accessed free at: http://genesdev.cshlp.org/content/22/6/810.full.html\#ref-list-1

\section{License}

Email Alerting Service right corner of the article or click here.
http://genesdev.cshlp.org/content/suppl/2008/03/03/22.6.810.DC1

Receive free email alerts when new articles cite this article - sign up in the box at the top

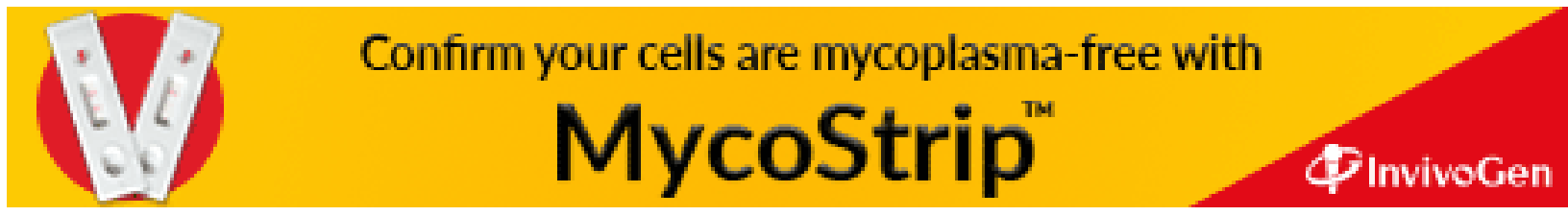

